NOTA CIENTIFICA

\title{
Mejoramiento de la calidad fustal en plantaciones de Pseudotsuga menziesii al crecer en asociación con Acacia melanoxylon
}

Improving the wood quality of Pseudotsuga menziesii growing in association with Acacia melanoxylon

\author{
HERBERT SIEBERT W. ${ }^{1}$, SOPHIE VON EINSIEDEL ${ }^{2}$, AGATHA FREIIN TRUCHSESS ${ }^{2}$ \\ ${ }^{1}$ Instituto de Silvicultura, Universidad Austral de Chile. E-mail: hsiebert49@hotmail.com
}

${ }^{2}$ Estudiante de la Facultad de Ciencias Forestales, Universidad de Göttingen, Alemania.

\begin{abstract}
SUMMARY
Site conditions in the south of Chile, from Cautín to Llanquihue $\left(38^{\circ} \mathrm{S}\right.$ to $\left.41^{\circ} \mathrm{S}\right)$, are well suited for Pseudotsuga menziesii crops. Despite 15,000 ha of $P$. menziesii plantations being planted to date, there is still little information on their management. To increase interest and research on this species, more acreage needs to be added and consideration given to high value wood production. At present, there are still doubts if the current management of P. menziesii in Chile will make possible the high value wood production required for international markets. Results in the present article demonstrate that the structure and volume of mixed $P$. menziesii stands have better characteristics for the production of higher value wood than pure stands. Furthermore, trees in mixed stands provide excellent continuous forest cover, a condition that would improve the long-term management of $P$. menziesii. The $P$. menziesiiA. melanoxylon association demonstrated a positive synergy, because A. melanoxylon decreased their bifurcation tendency while $P$. menziesii formed straight, uniform and self pruned stems.
\end{abstract}

Key words: Pseudotsuga menziesii, mixed plantations, stem quality.

\section{RESUMEN}

Las condiciones de sitio en el sur de Chile, de Cautín a Llanquihue $\left(38^{\circ}-41^{\circ} \mathrm{S}\right)$, ofrecen buenas perspectivas para el cultivo del Pseudotsuga mensiezii. A pesar de que a la fecha ya se cuenta con unas 15.000 ha de P. mensiezii en Chile, en su gestión quedan todavía muchas dudas por aclarar. Para hacer más interesante el trabajo con ella, es necesario aumentar considerablemente su superficie productiva y además considerar la producción de madera valiosa. Hoy en día aún existen dudas si con el esquema de manejo actualmente usado en Chile para $P$. menziesii sea posible producir maderas valiosas para el mercado internacional. Los resultados obtenidos del presente trabajo confirman que la estructura y el volumen de masas mixtas de $P$. menziesii poseen características más apropiadas para producir maderas valiosas que los rodales puros. Además, la estructura de masas mixtas presenta mejor predisposición para su conversión en cubiertas permanentes, lo cual mejoraría en el largo plazo la gestión con $P$. menziesii. La asociación P. menziesii-Acacia melanoxylon demostró una sinergia positiva, por cuanto A. melanoxylon disminuye su tendencia a la bifurcación y $P$. menziesii forma fustes más rectos, lisos y limpios de ramas.

Palabras claves: Pseudotsuga menziesii, plantaciones mixtas, calidad fustal. 


\section{INTRODUCCION}

Pseudotsuga menziesii o pino oregón es una conífera nativa de Norteamérica, altamente apreciada en el mercado internacional debido a las cualidades de su madera, su buena forma y crecimiento rápido (Infor 1998). Respecto de nuestro país, la especie llegó al sur de Chile a comienzos del 1900, pero recién a fines de los años 40 se realizaron los primeros cultivos comerciales, principalmente en la provincia de Cautín. Actualmente en el sur de Chile se cuenta con aproximadamente 15 mil hectáreas de P. menziesii (Infor 1998), la mayoría en forma de plantaciones puras con calidades regulares.

En el sur de Chile, provincias de Cautín, Valdivia, Osorno y Llanquihue, entre $\operatorname{los} 38^{\circ}$ a $42^{\circ}$ $\mathrm{S}$, se encuentran naturalmente en los sitios forestales productivos como especies principales Nothofagus obliqua, N. alpina y N. dombeyi. Coníferas, antaño de importancia comercial, como por ejemplo Araucaria araucana y Fitzroya cupressoides, se encuentran en sitios más extremos. Aquellas coníferas que crecen en sitios forestales productivos, bajo el dosel de los Nothofagus, como Podocarpus nubigenus, Saxegothaea conspicua y Podocarpus saligna, entre otras, presentan incrementos muy reducidos.

Como complemento en la práctica para el silvicultor puede ser de interés contar con una conífera de crecimiento rápido, de carácter intermedio, que se adapte e integre bien en los bosques naturales y que además posea buenas expectativas de mercado. La especie $P$. menziesii sin duda parece ser una buena alternativa (Siebert 1999).

En nuestro país, casi sin excepción, $P$. menziesii es cultivado de manera monoespecífica y es cosechado utilizando el método de tala rasa. Usual es la plantación sobre terreno descubierto. Desde el establecimiento, pasando por los cuidados culturales y hasta la cosecha, ocurre todo en extremo esquemático. Los árboles más vigorosos se desarrollan, casi sin excepción, hacia árboles lobos. Aquellos fustes con verticilos engrosados, con numerosas, gruesas y concentradas ramas con anillos anuales anchos, terminan en un mercado poco interesante en el transcurso de las cortas intermedias. Llegado el momento en que apenas un cierto número de árboles logra alcanzar diámetros aserrables, la masa completa es cortada utilizando el método de tala rasa, aun cuando aquellos indi- viduos de dimensiones menores son muy difíciles de comercializar.

Sin lugar a dudas, si se sigue utilizando este método ampliamente empleado en nuestro país para la especie Pinus radiata, no se lograrán porcentajes interesantes de madera estructural de buena calidad. Es así como frecuentemente surge la interrogante de cómo producir madera de $P$. menziesii para mercados más exigentes.

Esta circunstancia ha motivado el presente trabajo, el que pretende determinar las posibilidades de mejorar la calidad de los fustes de $P$. menziesii mediante el uso de la Silvicultura (Rittershofer 1994). Dentro de esta perspectiva, el presente trabajo tiene como objetivos mejorar la rectitud de fustes de $P$. menziesii, producción de fustes lisos (reducción de verticilos engrosados), mejorar su poda natural (reducción del número y grosor de ramas) y control del ancho de anillos anuales.

Los primeros ensayos para abordar esta problemática por la vía silvicultural comenzaron hace unos 15 a 20 años en la zona de este estudio. La tesis del trabajo surge al observar rodales de $P$. menziesii asociados con latifoliadas, los cuales dan la impresión de que esta especie presenta una mayor calidad fustal creciendo asociada. En masas puras llama especialmente la atención la gran cantidad de árboles con verticilos engrosados, que reducen considerablemente la calidad de los trozos (figura 1). Al aserrar trozos con verticilos en-

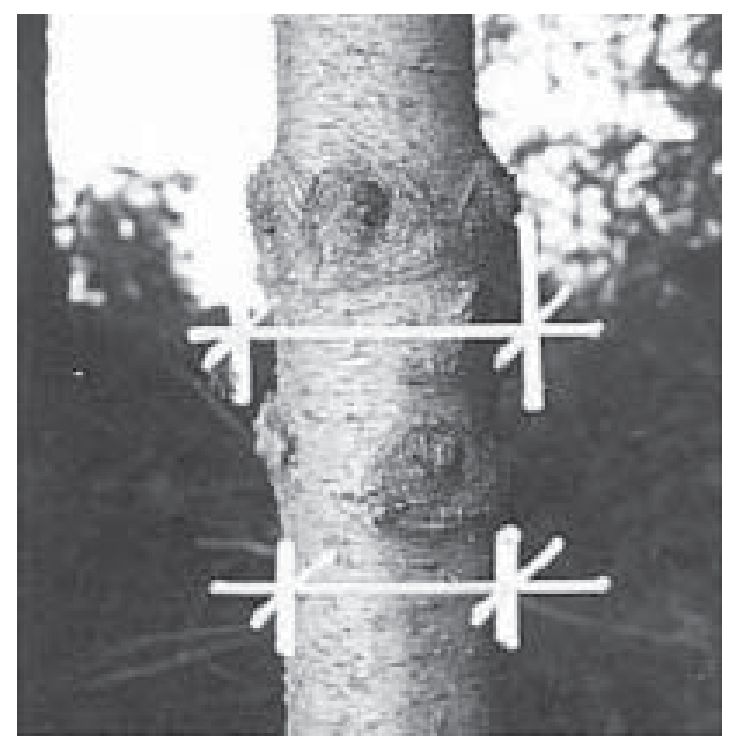

Figura 1: Verticilo engrosado de $P$. menziesii en masa pura.

P. menziesii enlarged verticiles in pure stands. 
grosados, la irregularidad del grano se proyecta a la madera aserrada. Por otra parte, P. menziesii creciendo bajo competencia con latifoliadas desarrolla una mejor calidad fustal para las necesidades de mercados más exigentes, con fustes largos, rectos, lisos libres de ramas y anillos anuales angostos.

\section{MATERIAL Y METODOS}

La zona donde se realizaron los ensayos fue el Fundo Miraflores (comuna de Lanco, provincia de Valdivia), que se extiende sobre una ladera norte del cordón de Trotrolhue, de aproximadamente $1.000 \mathrm{~m}$ de altitud. El fundo se extiende entre los 100 ms.n.m en el extremo norte hasta los 600 ms.n.m en el sur. Dominan suelos profundos de origen volcánico (trumaos), sobre roca metamórfica, caracterizados por poseer una alta porosidad (> 70\%), alta infiltración, buen drenaje y baja densidad. El suelo es rico en humus y posee una moderada disponibilidad de nutrientes. La precipitación anual fluctúa alrededor de los $2.000 \mathrm{~mm}$, bien repartidos en el año y sobre los 500 ms.n.m ocasionalmente nieve en invierno. Las temperaturas extremas van desde $\operatorname{los}-3^{\circ} \mathrm{C}$ a $\operatorname{los} 33^{\circ} \mathrm{C}$ con una media de $12^{\circ} \mathrm{C}$.

Las mediciones se realizaron en rodales puros de $P$. menziesii y mixtos de $P$. menziesii con $A$. melanoxylon. En los rodales mixtos ambas espe- cies crecen alternadas hilera por medio. Estas plantaciones se establecieron hace 13 a 16 años sobre pradera, con un espaciamiento de $3,4 \mathrm{~m}$ entre hileras y $1,8 \mathrm{~m}$ sobre la hilera.

En cada rodal muestreado se evaluaron 4 hileras puras de $P$. menziesii creciendo entre $A$. melanoxylon, en donde se midieron los parámetros a 50 árboles seguidos de $P$. menziesii (entre podados y no podados). En éstos se evaluó el engrosamiento de verticilos, cantidad de ramas por verticilo y rectitud de fuste. Como árboles con engrosamientos destacados se consideraron aquellos en los cuales el diámetro en el verticilo engrosado, a una altura entre 1,5 y $2 \mathrm{~m}$ del suelo, sobrepasa en un $20 \%$ o más al diámetro del fuste a esa altura. Además, en cada cuarto árbol se contó la cantidad de ramas por verticilo, para determinar si existe relación entre el número de ramas y los engrosamientos.

Los rodales muestreados se encuentran repartidos por todo el fundo, con diferentes exposiciones. Sus parámetros se observan en el cuadro 1 .

Mediciones. De las 1.635 plantas por hectárea establecidas originalmente $(1,8 \times 3,4 \mathrm{~m})$, se cuentan en estos momentos alrededor de 1.400. Por este motivo, las densidades consideradas para el análisis de datos son de 1.400 árboles por hectárea de $P$. mensiezii en rodales puros y 700 árboles por hectárea en los mixtos.

\section{CUADRO 1}

Parámetros de los rodales muestreados. Simple stand parameters

\begin{tabular}{|l|c|c|}
\hline \multicolumn{1}{|c|}{ Parámetros } & Masas puras de P. menziesii & Masas mixtas \\
\hline Edad media (años) & 15 & 13 \\
\hline Altura superior (m) & 11 & $\begin{array}{l}7(\text { P.m) } \\
\text { (A.m) }\end{array}$ \\
\hline Diámetro medio cuadrático (cm) & 18,22 & $13,2($ P.m) \\
\hline Densidad actual (N/ha) & 1.400 & $700($ P.m) \\
& & $700($ A.m) \\
\hline Area basal $\left(\mathrm{m}^{2} / \mathrm{ha}\right)$ & 38,5 & $10,8($ P.m) \\
\hline
\end{tabular}

P.m: Pseudotsuga menziessi; A.m: Acacia melanoxylon. 
BOSQUE 24(3): 75-83, 2003

Mejoramiento de la calidad fustal en plantaciones de Pseudotsuga menziesii al crecer en asociación...

\section{RESULTADOS Y DISCUSION}

Mientras en los rodales puros se muestra una distribución diamétrica normal (campana de Gauss), en las masas mixtas se observa una curva alargada con tendencia a una distribución heterodiamétrica (figura 2).

Engrosamientos y ramas. Las mediciones indican que en rodales puros los árboles de $P$. menziesii con verticilos engrosados constituyen un $42,7 \%$ de la masa total, mientras en los mixtos sólo un
$12,7 \%$ de los individuos de esta especie presentan engrosamientos (figuras 3 y 4).

Esta diferencia se debería principalmente a las distintas condiciones de luz en las cuales crece $P$. mensiezii. Mientras en los rodales puros los individuos de $P$. menziesii crecen expuestos a toda luz, en los rodales mixtos medidos crecen en la semisombra de los árboles de A. melanoxylon. Además, los individuos de $P$. menziesii con verticilos engrosados presentan entre 1,3 veces hasta el doble de ramas por verticilo comparado con aquellos sin engrosamientos (figura 5).

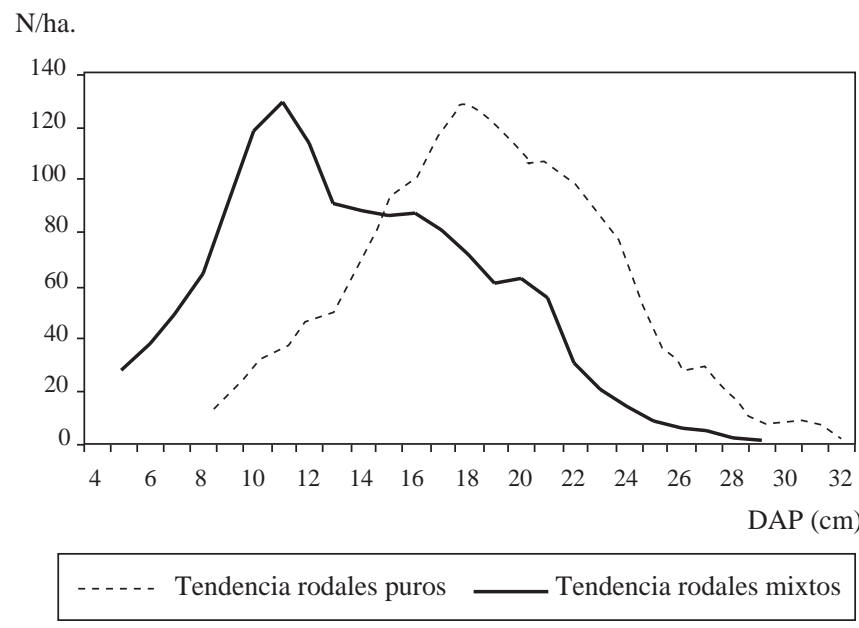

Figura 2: Curvas de distribución diamétrica de P. menziesii en masas puras y mixtas. $P$. menziesii diametrical distribution curves in mixed and pure stands.

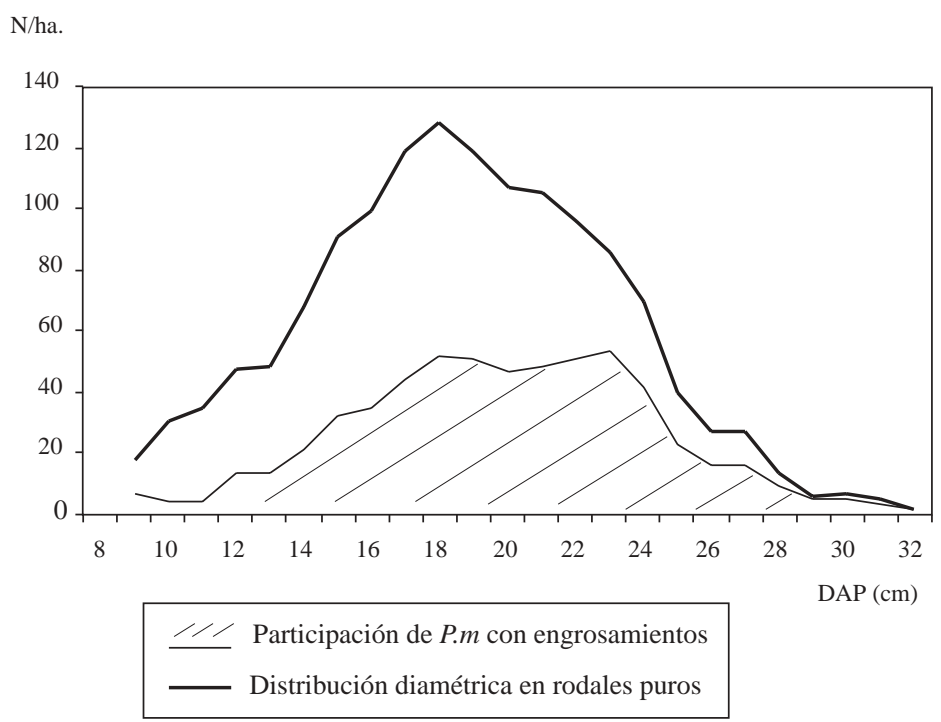

Figura 3: Participación de P. menziesii (P.m) con engrosamientos en rodales puros. $P$. menziesii (P.m) participation with enlarges in pure stands. 


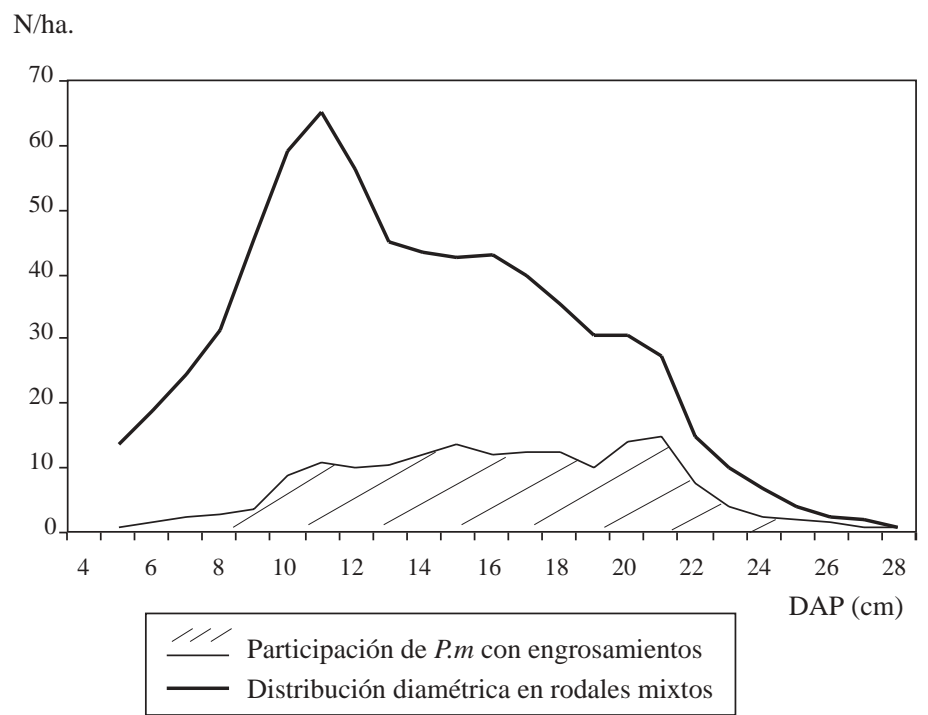

Figura 4: Participación de P. menziesii con engrosamientos en rodales mixtos. $P$. menziesii participation with enlarges in mixed stands.

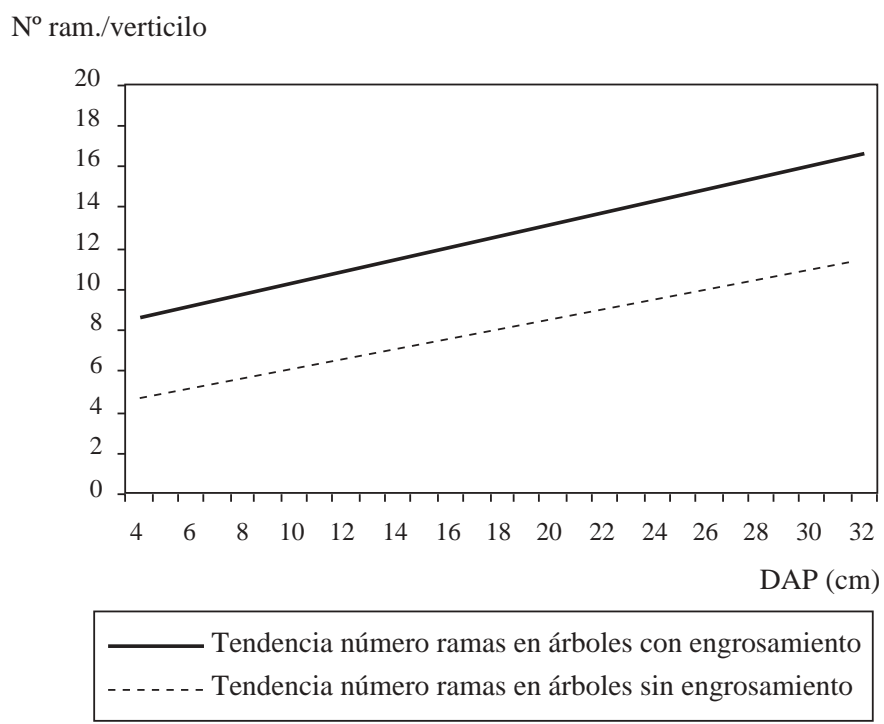

Figura 5: Comparación del número de ramas en árboles con y sin engrosamientos. Branch number comparison in trees with and without enlarges.

En las mediciones se observó, además, que en los individuos de $P$. menziesii con engrosamientos las ramas se ubican mucho más concentradas en los verticilos, lo cual en parte es una condición genética, pero acentuado por la influencia de la luz (figura 6). El P. menziesii, vigoroso, que crece a plena luz, desarrolla gruesas ramas y en él resaltan las características negativas de fuste deforme, engrosamientos, etc. En resumen, la plena luz transforma los árboles vigorosos de P. menziesii en árboles lobos. En cambio, creciendo en semisombra, el crecimiento del árbol vigoroso se mantiene controlado, y las características negativas no aparecen. Dicho de otra manera, la semisombra transforma los potenciales lobos en árbol selección, árbol porvenir o árbol "M".

Parece ser que la formación del engrosamiento es la reacción posterior del árbol para dar cabida a la gran cantidad de ramas gruesas concentradas, las cuales de lo contrario no tendrían cabida en el 
BOSQUE 24(3): 75-83, 2003

Mejoramiento de la calidad fustal en plantaciones de Pseudotsuga menziesii al crecer en asociación...

verticilo. Dengler (1982) sostiene, en cambio, que los engrosamientos son producidos por la cicatrización después de la poda de ramas gruesas.

Presencia de P. menziesii potenciales $M$. Como árboles potenciales "M" o de madurez, se entienden aquellos árboles de selección (Schädelin 1936) que a través del manejo de la masa presumiblemente llegarán a la madurez y serán cortados al alcanzar su diámetro objetivo ("Z-Bäume" de Schädelin 1936).

Para caracterizar la calidad o el potencial de los rodales para producir fustes valiosos se seleccionaron individuos de $P$. menziesii $\mathrm{M}$ y/o potenciales $\mathrm{M}$. Aquellos árboles potenciales $\mathrm{M}$ de $P$. menziesii presentaban fustes rectos, lisos, con ramas finas y diseminadas, como se puede observar en la figura 7. Los rodales de P. menziesii puros y mixtos se compararon de acuerdo al porcentaje de árboles potenciales M. En masas puras, sólo un $27,3 \%$ de los individuos de $P$. menziesii son potenciales $\mathrm{M}$, mientras en los rodales mixtos lo son un $52,3 \%$, lo cual se puede observar en las figuras 8 y 9 .

En las masas puras los individuos potenciales $\mathrm{M}$ de $P$. menziesii presentan un DMC de $16,5 \mathrm{~cm}$, siendo este incremento en diámetro un $30 \%$ más alto que el de los individuos potenciales $\mathrm{M}$ en rodales mixtos, los cuales alcanzan un DMC de $11,8 \mathrm{~cm}$. Sin embargo, si entre los objetivos de producción o manejo además se considera producir maderas de anillos anuales angostos, las masas mixtas presentan mejores expectativas (cuadro 2). Para $P$. menziesii mercados exigentes fijan un mínimo de tres anillos de crecimiento anual por centímetro (3,3 $\mathrm{mm}$ por anillo).

La figura 10 muestra que mientras en las clases diamétricas superiores las distribuciones de los $P$. menziesii potenciales $\mathrm{M}$ en rodales puros y mixtos son casi coincidentes, las masas mixtas poseen adicionalmente una gran reserva de $P$. menziesii potenciales $\mathrm{M}$ en las clases diamétricas inferiores para el futuro. Los aproximadamente 200 individuos de $P$. menziesii potenciales $\mathrm{M}$ de estas clases diamétricas inferiores en masas mixtas presentan un ancho medio de anillos de alrededor de 3,3 $\mathrm{mm}$, con lo cual se estaría dando cumplimiento a las exigencias mencionadas para los mercados exigentes.

En los rodales mixtos también se evaluó la calidad fustal de la especie asociada A. melanoxylon.

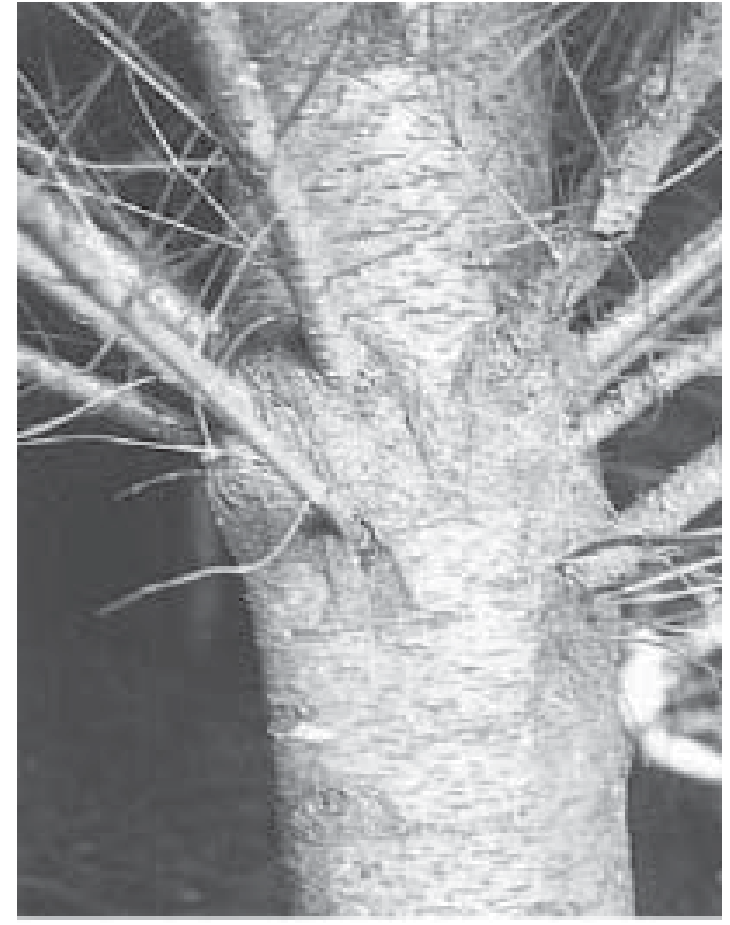

Figura 6: Verticilos con ramas concentradas. Concentrated branch verticiles.

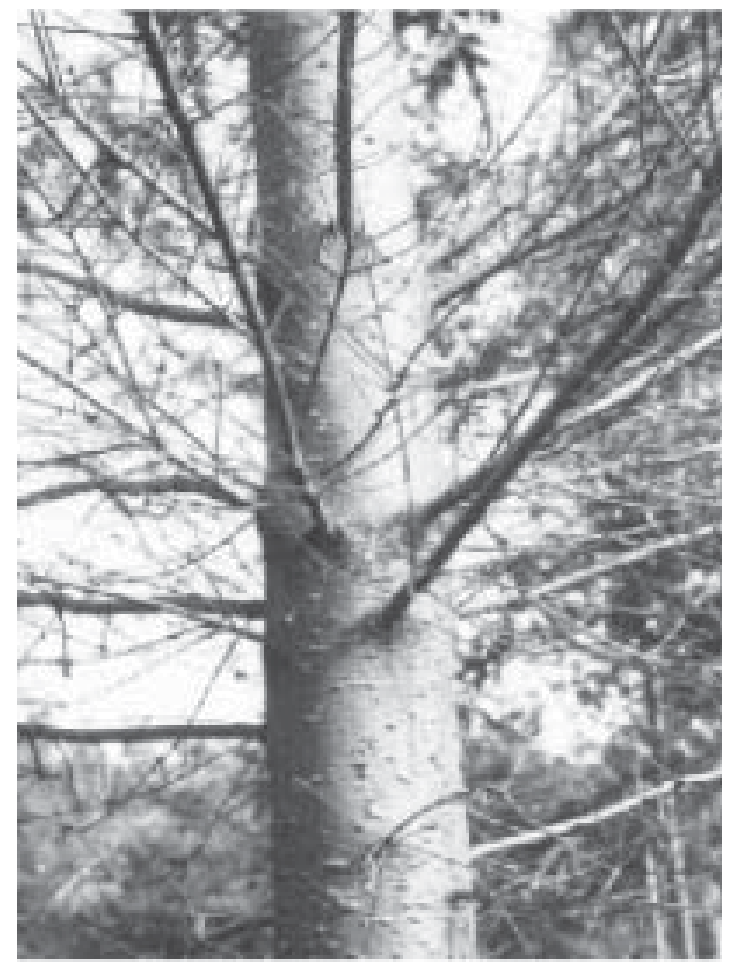

Figura 7: Verticilos con ramas diseminadas. Disseminated branch verticiles. 
N/ha

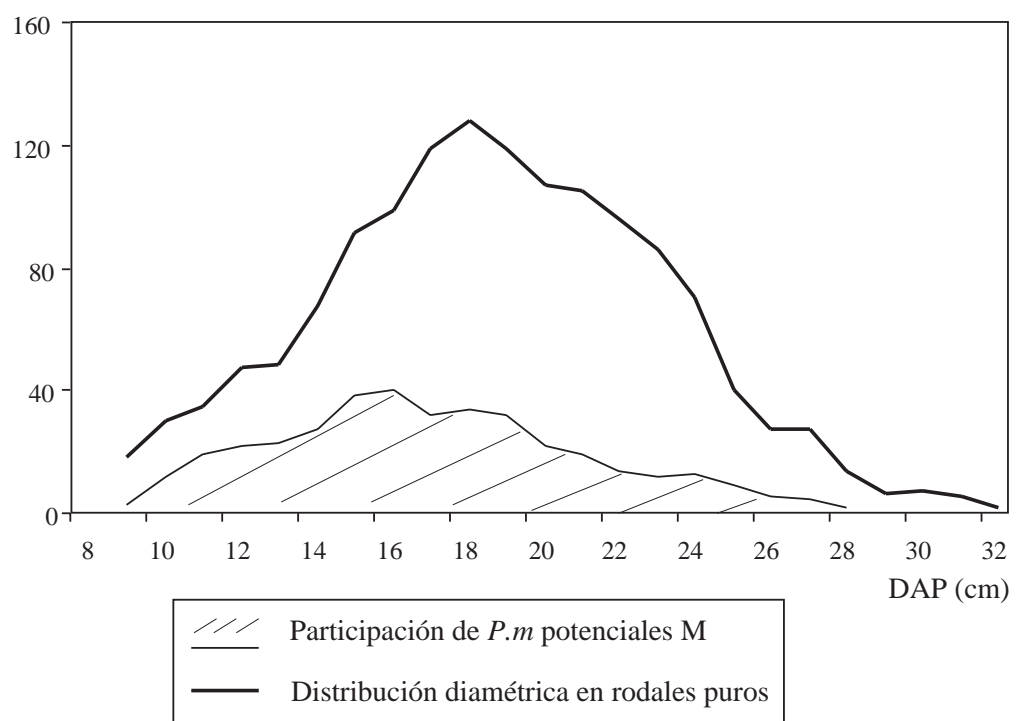

Figura 8: Participación de individuos de P. menziesii (P.m) potenciales M en rodales puros.

P. menziesii (P.m) potential and participation in pure stands.

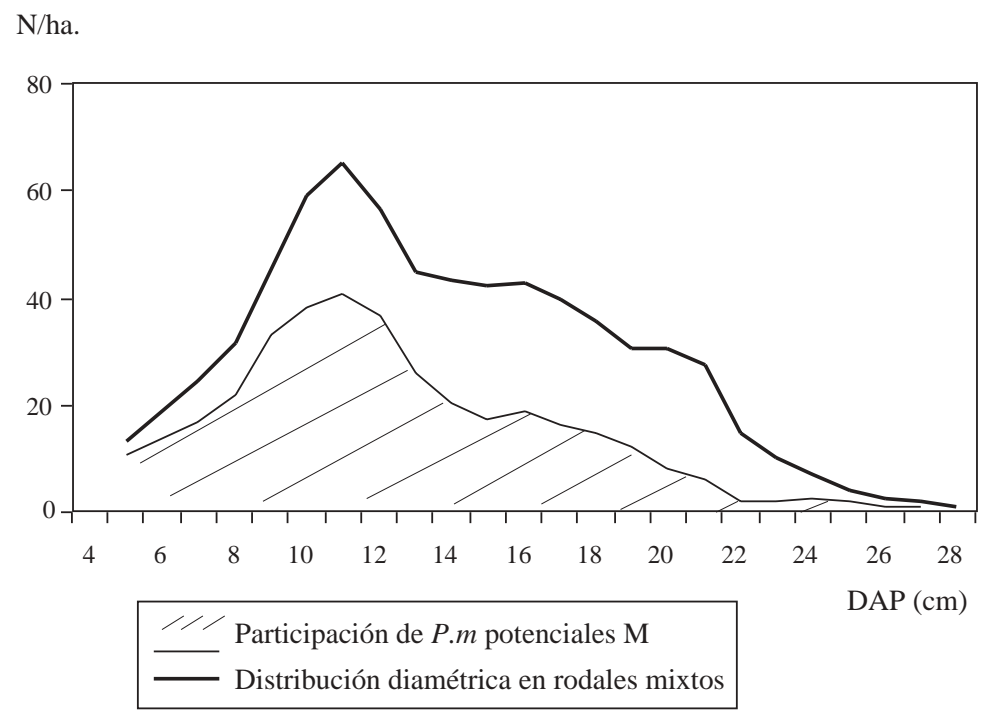

Figura 9: Participación de individuos de P. menziesii (P.m) potenciales $\mathrm{M}$ en rodales mixtos.

P. menziesii (P.m) potential M and participation in mixed stands.

Las experiencias indican que el principal problema de esta especie es la bifurcación del fuste, ya que los individuos bifurcados corren un gran riesgo de quebrarse con el viento o la nieve. En masas puras de A. melanoxylon se determinó que sobre un $95 \%$ de los árboles presentan bifurca- ciones en el fuste, y por lo tanto no sirven como árboles $\mathrm{M}$.

En masas asociadas con $P$. menziesii se puede concluir, a través de diferentes mediciones realizadas, que entre un 30 a un $40 \%$ de los individuos de A. melanoxylon presentan crecimiento monopódico. 
BOSQUE 24(3): 75-83, 2003

Mejoramiento de la calidad fustal en plantaciones de Pseudotsuga menziesii al crecer en asociación...

\section{CUADRO 2}

Tendencia del ancho de anillos anuales en masas puras y mixtas, y a su vez en

$P$. menziesii con engrosamientos y $P$. menziesii potenciales $\mathrm{M}$.

Annual ring width tendencies in pure and mixed stands.

\begin{tabular}{|l|c|c|c|}
\cline { 2 - 4 } \multicolumn{1}{c|}{} & $\begin{array}{c}\text { P. menziesii } \\
\text { con engrosamiento } \\
\text { (mm/año) }\end{array}$ & $\begin{array}{c}\text { Todos los árboles } \\
\text { medidos } \\
(\mathrm{mm})\end{array}$ & $\begin{array}{c}\text { P. menziesii } \\
\text { potenciales M } \\
(\mathrm{mm})\end{array}$ \\
\hline Puros & 6,5 & 6,1 & 5,5 \\
\hline Mixtos & 6,2 & 5,5 & 5,1 \\
\hline
\end{tabular}

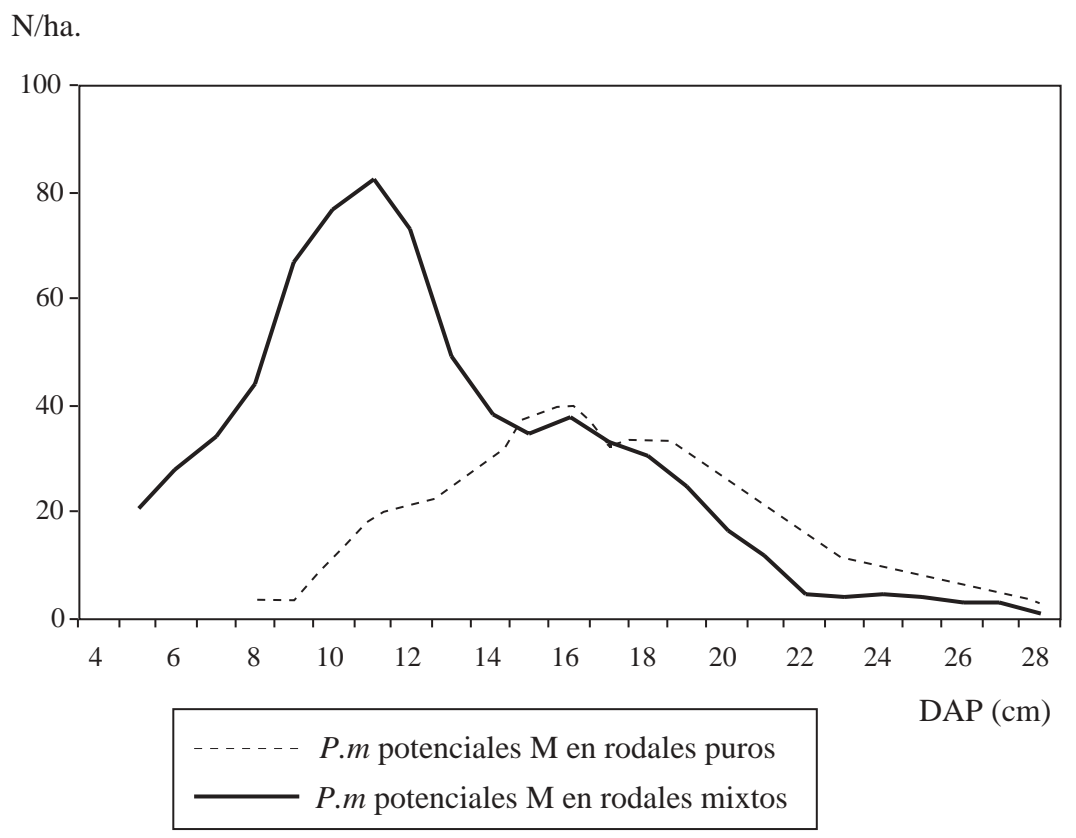

Figura 10: Comparación en la distribución diamétrica de individuos de P. menziesii (P.m) potenciales $\mathrm{M}$ en rodales puros y mixtos.

P. menziesii (P.m) potential and diametrical distribution comparison in pure and mixed stands.

\section{CONCLUSIONES}

Si se desea producir madera de $P$. menziesii para mercados exigentes, a precios interesantes, será muy difícil lograrlo de manera satisfactoria en plantaciones puras creciendo a plena luz. Cultivando $P$. menziesii en su juventud en semisombra se lograrán fustes rectos, lisos, de ramas delgadas y anillos de crecimiento angostos.
Al asociar P. menziesii con A. melanoxylon se logran condiciones ventajosas para ambas especies. Para P. menziesii, por cuanto A. melanoxylon crece más rápido en altura, aportando a $P$. menziesii la semisombra que mejora su calidad y, para $A$. melanoxylon significa aumentar la cantidad de ejemplares de crecimiento monopódico, necesarios para el mercado de maderas valiosas. 
BOSQUE 24(3): 75-83, 2003 Mejoramiento de la calidad fustal en plantaciones de Pseudotsuga menziesii al crecer en asociación...

\section{DEDICATORIA}

Este trabajo está dedicado a Don Karl Weber K., pionero en la gestión comercial de $P$. menziesii en Chile.

\section{BIBLIOGRAFIA}

DENGLER, A. 1982. Waldbau auf ökologischer Grundlage, neubearbeitet von I. Bonnemann, Band 1 u. 2, 5. Auflage, Hamburg/Berlin. 283 p
INSTITUTO FORESTAL 1998. Monografía de Pino oregón (Pseudotsuga menziesii). Proyecto Potencialidad de especies y sitios para una diversificación silvícola nacional. $143 \mathrm{p}$.

RITTERSHOFER, F. 1994. Waldpflege und Waldbau. Rittershofer Verlag, Freising. 481 p.

SCHÄDELIN, W. 1936. Die Durchforstung als Auslese- und Veredelungsbetrieb höchster Wertleistung. En: Heyder, Chr. Waldban im Wendel J.D. Sanerländer's Verlag, 1986, Frankfurt, $602 \mathrm{p}$.

SIEBERT, H. 1999. La Silvicultura Alternativa: Un concepto silvícola para el bosque nativo chileno. En: Silvicultura de los bosques nativos de Chile. Ed. Universitaria, Santiago. 381-406 